\title{
NOTCH Signaling Is Activated through Mechanical Strain in Human Bone Marrow-Derived Mesenchymal Stromal Cells
}

\author{
Fani Ziouti, ${ }^{1}$ Regina Ebert $\mathbb{D}^{2},{ }^{2}$ Maximilian Rummler, ${ }^{3}$ Melanie Krug, ${ }^{2}$ \\ Sigrid Müller-Deubert $\mathbb{D}^{\mathbb{C}},{ }^{2}$ Martin Lüdemann, ${ }^{2}$ Franz Jakob, ${ }^{2}$ Bettina M. Willie, ${ }^{3}$ \\ and Franziska Jundt $\mathbb{D}^{1}$ \\ ${ }^{1}$ Department of Internal Medicine II and Comprehensive Cancer Center Mainfranken, University Hospital of Würzburg, \\ Oberdürrbacher Straße 6, 97080 Würzburg, Germany \\ ${ }^{2}$ Orthopedic Center for Musculoskeletal Research, University of Würzburg, Friedrich-Bergius-Ring 15, 97076 Würzburg, Germany \\ ${ }^{3}$ Research Centre, Shriners Hospital for Children-Canada, Department of Pediatric Surgery, McGill University, 1003 Decarie Blvd, \\ Montreal, Canada H4A OA9
}

Correspondence should be addressed to Franziska Jundt; Jundt_F@ukw.de

Fani Ziouti and Regina Ebert contributed equally to this work.

Received 6 August 2018; Accepted 28 October 2018; Published 26 February 2019

Academic Editor: Susanna Miettinen

Copyright (C) 2019 Fani Ziouti et al. This is an open access article distributed under the Creative Commons Attribution License, which permits unrestricted use, distribution, and reproduction in any medium, provided the original work is properly cited.

\begin{abstract}
Skeletal development and remodeling of adult bone are critically controlled by activated NOTCH signaling in genetically modified mice. It is yet unclear whether NOTCH signaling is activated by mechanical strain sensed by bone cells. We found that expression of specific NOTCH target genes is induced after in vivo tibial mechanical loading in wild-type mice. We further applied mechanical strain through cyclic stretching in human bone marrow-derived mesenchymal stromal cells (BMSCs) in vitro by using a bioreactor system and detected upregulation of NOTCH target gene expression. Inhibition of the NOTCH pathway in primary BMSCs as well as telomerase-immortalized human BMSCs (hMSC-TERT) through the gamma-secretase inhibitor GSI XII blocked mechanotransduction and modulated actin cytoskeleton organization. Short-hairpin RNA gene silencing identified NOTCH2 as the key receptor mediating NOTCH effects on hMSC-TERT cells. Our data indicate a functional link between NOTCH activation and mechanotransduction in human BMSCs. We suggest that NOTCH signaling is an important contributor to molecular mechanisms that mediate the bone formation response to mechanical strain.
\end{abstract}

\section{Introduction}

The NOTCH signaling pathway is evolutionarily highly conserved and regulates cell growth, cell death, and differentiation programs via cell-cell communication [1]. NOTCH receptors (NOTCH1-4) on receiving cells are activated through ligands (JAGGED (JAG1, JAG2) and DELTA-like (DLL1, DLL3, and DLL4)) binding on neighboring cells [1]. After a proteolytic cleavage cascade, the intracellular part of the receptor (NIC) is cleaved involving a $\gamma$-secretase enzyme activity, which can be blocked by $\gamma$-secretase inhibitors (GSI) [1]. NIC translocates to the nucleus, binds to recombination signal-binding protein for immunoglobulin kappa J region
(RBPjk) and mastermind-like (MAML) proteins in a complex, and controls transcription of canonical NOTCH target genes: the hairy and enhancer of split HES1 and HES-related with YRPW motif HEY1-, HEY2-, and HEY-like (HEYL) gene families [1].

Conflicting data of studies with global NOTCH deletion in mice mainly reflect the apparent cell- and stage-specific function of NOTCH during skeletal development [1]. In young mice, NOTCH signaling maintains the pool of bone marrow-derived mesenchymal stromal cells (BMSCs), the skeletal precursors [2]. In contrast, deletion of members of the NOTCH pathway stimulates osteogenic differentiation and trabecular bone formation early on [2], but with aging, 
the BMSC pool in these knockout mice is diminished, resulting in an osteopenic phenotype. Osteopenia is exacerbated by an overproduction of the osteoclast-stimulating receptor activator of NF-kappaB ligand (RANKL) in mature osteoblasts [2]. One important downstream effector mechanism in this context may be the inhibitory effects of NOTCH target genes HES1 and HEY1 on the osteogenic commitment of skeletal precursors, which suppress the transcriptional activity of the core osteogenic transcription factor RUNX2 and the expression of downstream osteogenic marker genes [1]. Conditional overexpression of $\mathrm{NOTCH}$ in the osteoblastic lineage at various differentiation stages confirms NOTCH's role in maintaining the early differentiation stage of BMSCs. However, conflicting roles of NOTCH signaling in osteocyte development and function were reported: (1) Overexpression of $\mathrm{NOTCH}$ in mature osteocytes increases bone formation due to an induction of osteoprotegerin (OPG) production and a diminished secretion of the WNT inhibitors sclerostin (SOST) and dickkopf 1 (DKK1). This results in enhanced osteogenic canonical WNT signaling, which is coincident with suppressed bone resorption [3,4]. (2) Data in vitro show that during the transition phase from osteoblasts to osteocytes, a crosstalk between NOTCH and canonical WNT signaling is observed leading to WNT signaling inhibition [5]. Vice versa, osteocyte-specific overexpression of $\beta$-catenin and subsequent activation of WNT signaling result in increased NOTCH signaling in the bone microenvironment of mice [6].

Active NOTCH further controls bone remodeling processes in mice. Cell-specific activation of NOTCH signaling has anabolic actions on mature bones, promotes bone healing, and prevents tamoxifen-induced bone loss [7]. The transient use of GSI in a murine model of skeletal fracture repairs accelerated bone and cartilage formation via promotion of callus formation and BMSC differentiation, which is coincident with a slight reduction in BMSC numbers [8].

So far, it is unknown whether NOTCH signaling is involved in mechanotransduction in human BMSCs as precursors of human osteoblasts and osteocytes, which may be relevant during fracture healing and bone regeneration. Mechanotransduction as a response to cyclic stretching or fluid flow determines cell fate decision, lineage commitment, and differentiation of mesenchymal precursors. Mechanical loads applied to the bone are converted into a local mechanical signal (widely thought to be strain) that is sensed by bone cells resulting in biochemical cues by use of mechanoreceptors, such as cell membrane-associated proteins, e.g., integrins or calcium channels. As a result, mechanoresponsive signaling cascades such as the mitogen activated protein kinase (MAPK) pathway, which translate mechanical signals into the activation of transcription factors such as AP-1 or SP1, are activated. Both factors bind to responsive elements in the nuclei of effector cells [9-12].

There are hints from the literature that NOTCH signaling might be mechanosensitive and might also influence mechanosensitivity and downstream mechanoresponse. It was shown in a zebrafish model that $\mathrm{NOTCH}$ receptor and target gene expression is activated after the application of hemodynamic shear stress during cardiomyocyte trabeculation [13]. With a tension gauge tether assay and by immobilizing the NOTCH ligands JAG1 or DLL1 to a surface, it has been demonstrated that NOTCH signaling is activated by force application in yeast and in $\mathrm{NOTCH} 1$ overexpressing $\mathrm{CHO}-\mathrm{K} 1$ cells, respectively [14]. Angiogenesis and osteogenesis are defective when blood flow is impaired in vessels of murine long bones, which is coincident with downregulated NOTCH signaling in endothelial cells. Artery formation could be rescued by the overexpression of the active NOTCH1 intracellular domain, and it has been shown that NOTCH signaling controls the expression of fluid flow-responsive genes in endothelial cells and modulates the formation of fluid flow-sensing primary cilia [15-18].

In this study, we detect NOTCH activation in bone cells after in vivo tibial mechanical loading in mice and after cyclic stretching of human BMSCs in vitro by use of a small-scale cell culture/bioreactor system.

\section{Materials and Methods}

2.1. In Vivo Mechanical Loading. RNA was received from wild-type littermate control mice, used in the recently published study by Pflanz et al. [19]. Briefly, the left tibiae of six 10-week-old female C57BL/6 mice underwent a single bout of in vivo cyclic compressive loading $(216$ cycles at $4 \mathrm{~Hz}$, peak strains at a tibial midshaft of $+900 \mu \varepsilon$ ) while under anesthesia. Mice were sacrificed at 1 or $24 \mathrm{~h}$ after the single loading session, and the right and left tibiae were extracted. Bone marrow was flushed, and RNA was isolated from osteocyte-enriched hard tissue of both limbs using the TRIzol ${ }^{\circledR}$ reagent (Thermo Fisher Scientific, Darmstadt, Germany) followed by purification with an RNeasy kit (QIAGEN GmbH, Hilden, Germany) as described previously [19]. Total RNA was used for first-strand cDNA synthesis with the iScript ${ }^{\mathrm{TM}}$ cDNA Synthesis Kit (Bio-Rad Laboratories, Hercules, USA) according to the manufacturer's instructions. Real-time quantitative PCR (qPCR) was performed with the 2x SYBR Green qPCR Master Mix (Bimake, Houston, USA) on a QuantStudio 7 (Thermo Fisher Scientific, Darmstadt, Germany) system. Each reaction was performed in technical duplicate, $\mathrm{Ct}$ values were averaged, and relative gene expression was calculated as previously described. Right tibiae served as internal nonloaded controls and were used for normalization. GAPDH served as a housekeeping gene. As was previously reported [19], the animal experiments were carried out according to the policies and procedures approved by the local legal research animal welfare representative (LaGeSo, Berlin, G0021/11).

2.2. Cell Culture. Primary human BMSCs were obtained from the femoral head of 12 different donors $(5$ males, 7 females, mean age $63.5 \pm 12.6)$ undergoing elective hip arthroplasty. Material was collected with informed consent from all patients, and the procedure was approved by the local Ethics Committee of the University of Würzburg (06/30/2010). In brief, bone marrow was washed with Dulbecco's modified Eagle's medium (DMEM/F12) (Thermo Fisher Scientific, Darmstadt, Germany) supplemented with $10 \%$ fetal calf serum (Bio\&Sell GmbH, Feucht, Germany) [20], $100 \mathrm{U} / \mathrm{ml}$ penicillin, $0.1 \mathrm{mg} / \mathrm{ml}$ streptomycin, and $50 \mu \mathrm{g} / \mathrm{ml}$ ascorbate (Sigma-Aldrich GmbH, Munich, Germany) and centrifuged 
at $1200 \mathrm{rpm}$ for $5 \mathrm{~min}$. Pellet was washed four times with complete medium, and resulting supernatants containing released cells were collected. Cells were pelleted and cultured at a density of $1 \times 10^{9}$ cells per $175 \mathrm{~cm}^{2}$ culture flask. After 2 days, nonattached cells were washed away and adherent ones were cultivated until confluence [21]. HMSC-TERT cells were established from a 33-year-old male donor by the group of Kassem (Odense, Denmark). An AP-1 response element was cloned into the pGL4.14 luciferase reporter vector (Promega, Mannheim, Germany), and the stable hMSC-TERTAP-1 cell line was generated by electroporation [22]. AP-1 response elements have been shown to be activated by mechanical stimuli. Therefore, the hMSC-TERT-AP-1 clone was used as a tool to perform mechanistic studies. Cells comprising the empty pGL4.14 vector served as controls. HMSC-TERT cells show high proliferation rates, while maintaining their mesenchymal differentiation capacity in vitro and in vivo $[23,24]$. HMSC-TERT-AP-1 cells were cultured in Eagle's MEM supplemented with $10 \%$ FCS and $50 \mu \mathrm{g} / \mathrm{ml}$ hygromycin. 293T cells (DSMZ, Braunschweig, Germany) were cultured in Dulbecco's modified Eagle's medium (Thermo Fisher Scientific, Darmstadt, Germany) supplemented with $10 \% \mathrm{FCS}, 100 \mathrm{IU} / \mathrm{ml}$ penicillin, and $100 \mu \mathrm{g} / \mathrm{ml}$ streptomycin. All cells were cultivated at $37^{\circ} \mathrm{C}$ in a humidified 95\% air and $5 \% \mathrm{CO}_{2}$ atmosphere.

2.3. Lentiviral Transfer of shRNAs for Knockdown of NOTCH1 and NOTCH2 in hMSC-TERT Cells. Lentiviral particles containing short-hairpin RNAs (shRNAs) (designed

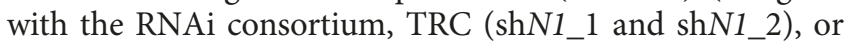
obtained from the literature (shN2) [25] for knockdown of NOTCH1 and NOTCH2) were generated by co-transfection of the pLKO (shN1_1 and shN1_2) or the pGIPZ (sh2) vector and the packaging plasmids psPAX.2 and pMD2.G into 293T cells. Plasmids were obtained from Addgene (Teddington, UK) and Dharmacon (pGIPZ, Lafayette, USA). In brief, plasmids were mixed with Opti-MEM (Thermo Fisher Scientific, Darmstadt, Germany) and the transfection reagent polyethylenimine (Sigma-Aldrich $\mathrm{GmbH}$, Munich, Germany) and added to 293T cells with 2\% FCS-supplemented media overnight. Complete media replaced the transfection mix the next day. Supernatants with virus particles were harvested over two days, filtered, and stored at $-80^{\circ} \mathrm{C} .6 \times 10^{5} \mathrm{hMSC}-\mathrm{TERT}$ AP-1 cells were transduced by adding virus supernatants, complete media, and hexadimethrine bromide (Sigma-Aldrich $\mathrm{GmbH}$, Munich, Germany), and $24 \mathrm{~h}$ later, complete media replaced the infection mix. Selection with puromycin (Thermo Fisher Scientific, Darmstadt, Germany) started after $24 \mathrm{~h}$ and lasted for 3 days. Lentiviral particles with empty vectors or vector expressing nontargeting shRNA were used as controls. Knockdown in transduced cells was confirmed by qPCR for NOTCH1 and NOTCH2. shRNA target sequences were as follows: GAACGAGCATAGTCCA AAAA (shN1_1), GTAGTTGTTCGTTGGTTATA (shN1_ 2), GCCCAATGTCTCTTGTGACATA $(\operatorname{sh} N 2)$, and TCTC GCTTGGGCGAGAGTAAG (non-targeting control).

2.4. Immunoblotting. Whole protein lysates were prepared as previously described [26]. Briefly, $100 \mu \mathrm{l}$ lysis buffer $(50 \mathrm{mM}$
Tris $\mathrm{pH} 7.5,150 \mathrm{nN} \mathrm{NaCl}, 1 \%$ Triton, $0.5 \% \mathrm{NP} 40$, and $0.2 \%$ SDS) containing protease inhibitors (Protease Inhibitor Cocktail, Sigma-Aldrich GmbH, Munich, Germany) was used, and protein concentrations were determined using the Roti ${ }^{\circledR}$-Quant reagent (Carl Roth GmbH, Karlsruhe, Germany). $30 \mu \mathrm{g}$ protein was mixed with $5 \mu \mathrm{l}$ loading buffer (375 mM Tris- $\mathrm{HCl}$ pH 6.8, 6\% SDS, 4.8\% glycerol, 9\% 2-mercaptoethanol, and $0.03 \%$ bromophenol blue) and denatured at $95^{\circ} \mathrm{C}$ for $5 \mathrm{~min}$. Proteins were separated on $10 \%$ polyacrylamide (SDS-PAGE) gels in $0.3 \%$ Tris, $0.144 \%$ glycin, and $0.5 \%$ SDS buffer and transferred to Whatman ${ }^{\mathrm{TM}}$ Protran $^{\mathrm{TM}}$ Nitrocellulose Blotting Membranes (Thermo Fisher Scientific, Darmstadt, Germany) at $1.5 \mathrm{~mA} / \mathrm{cm}^{2}$ of membrane for $2 \mathrm{~h}$. Membranes were blocked with 5\% SDS in TBS-T buffer (50 mM Tris, $150 \mathrm{mM} \mathrm{NaCl}$, and $0.1 \%$ Tween-20) and incubated overnight at $4{ }^{\circ} \mathrm{C}$ with the following primary antibodies against human epitopes: rabbit anti-NOTCH1 (1:1000, Cell Signaling Technology, Frankfurt am Main, Germany), rabbit anti-NOTCH2 (1:1000, Cell Signaling Technology, Frankfurt am Main, Germany), mouse anti-HES1 (1:2500, Santa Cruz Biotechnology, Heidelberg, Germany), and mouse anti-vinculin (1:10000, Sigma-Aldrich GmbH, Munich, Germany). Membranes were washed 3 times for 5 min with TBS-T and incubated for $1 \mathrm{~h}$ at room temperature with horseradish peroxidase-conjugated anti-rabbit and anti-mouse IgG (all from Cell Signaling Technology, Frankfurt am Main, Germany) secondary antibodies. After another washing 3 times for $5 \mathrm{~min}$ with TBS-T, proteins were visualized using the SuperSignal ${ }^{\mathrm{TM}}$ West Dura Extended Duration Substrate (Thermo Fisher Scientific, Darmstadt, Germany) and detection was performed on a ChemiDoc MP Imaging System (Bio-Rad Laboratories, Hercules, USA) using the Image Lab Software (version 5.2.1, Bio-Rad Laboratories, Hercules, USA). All bands were densitometrically analyzed with ImageJ [27].

2.5. Cell Viability and Apoptosis Assays. To determine the effects of the NOTCH inhibitor GSI XII (Merck, Darmstadt, Germany) on viability and apoptosis, BMSCs and hMSC-TERT cells were seeded on a 96-well plate with a density of 1000 cells/well. Cells were treated with 2 or $4 \mu \mathrm{M}$ GSI XII or the solvent DMSO $(0 \mu \mathrm{M})$ for 24 and $48 \mathrm{~h}$. Viability and apoptosis rates were assessed using the CellTiter-Glo Luminescent Cell Viability Assay and the Caspase-Glo 3/7 Assay, respectively (both from Promega GmbH, Mannheim, Germany), according to the manufacturer's instructions. Luminescence was measured with an Orion II Luminometer (Berthold Detection Systems, Pforzheim, Germany). Data are expressed as mean from triplicates of three independent experiments (hMSC-TERT) or five independent donors (BMSC).

2.6. Cyclic Stretching of hMSC-TERT-AP-1 Cells and Luciferase Assay. $3 \times 10^{4}$ cells per well were seeded on 24-well polyurethane (PU) plates, and tight cell attachment was observed few hours later, as shown before [22]. After $24 \mathrm{~h}$, cells were treated with the solvent DMSO (indicated as $0 \mu \mathrm{M})$ or $2 \mu \mathrm{M}$ and $4 \mu \mathrm{M}$ GSI XII, and after incubation for $24 \mathrm{~h}, \mathrm{PU}$ dishes were placed in a bioreactor [22]. Cyclic 
stretching was applied twice for $30 \mathrm{~min}(1 \mathrm{~Hz}$ and $1 \%$ extension), with a $60 \mathrm{~min}$ pause in between. After $24 \mathrm{~h}$, cells were lysed in $150 \mu \mathrm{l}$ Reporter Lysis Buffer (Promega GmbH, Mannheim, Germany) and $20 \mu \mathrm{l}$ of extracts was used for the measurement of luciferase activity with the reporter gene assay (Promega GmbH, Mannheim, Germany) in an Orion II Luminometer (Berthold Detection Systems, Pforzheim, Germany). Protein content was determined with the Roti-Quant Protein Assay (Carl Roth GmbH, Karlsruhe, Germany) and used for normalization of luminescence units. Four technical replicates were obtained from four independent wells.

2.7. Cyclic Stretching of BMSCs. BMSCs were obtained from different donors as indicated, and $5 \times 10^{5}$ cells per well were seeded on 4-well PU plates, allowed to attach, and cultured for one week. Cells were treated with 2 and $4 \mu \mathrm{M}$ GSI XII or the solvent DMSO $(0 \mu \mathrm{M})$ for $24 \mathrm{~h}$. PU dishes were placed in a bioreactor, and cyclic stretching was applied as previously described $[10,12] .15 \mathrm{~min}$ and $4 \mathrm{~h}$ later, cells were harvested and total RNA was isolated by using the NucleoSpin RNA II kit (Macherey-Nagel, Düren, Germany) according to the manufacturer's instructions.

2.8. Reverse Transcription and $q P C R$ Analysis. For mRNA reverse transcription, one microgram of total RNA was used for first-strand cDNA synthesis with MMLV reverse transcriptase (Promega GmbH, Mannheim, Germany) in $25 \mu \mathrm{l}$ total volume as previously described [28]. cDNA was diluted to $1: 10$, and $2 \mu \mathrm{l}$ was used for real-time qPCR with the GoTaq qPCR Master Mix (Promega GmbH, Mannheim, Germany) in a $20 \mu \mathrm{l}$ total volume. Sequence-specific primers (5 pmol per reaction) were designed using the Universal ProbeLibrary System Assay Design (Roche, Mannheim, Germany). Primers were obtained from QIAGEN GmbH (Hilden, Germany) or from published studies as indicated (see Table 1 for primer sequences and PCR conditions). Each reaction was performed in technical triplicate, and $\mathrm{Ct}$ values were averaged. Relative gene expression was calculated with the efficiency-corrected Ct model [29] with RPS27A as the housekeeping gene [30].

2.9. Actin Cytoskeleton Staining with Phalloidin. 2000 BMSCs per $\mathrm{cm}^{2}$ were seeded on 12-well plates and grown overnight. Cells were treated with 2 or $4 \mu \mathrm{M}$ GSI XII or the solvent DMSO for 24 or $48 \mathrm{~h}$, respectively, washed with PBS, and fixed with $4 \%$ paraformaldehyde for $10 \mathrm{~min}$. After three additional PBS washing steps, cells were incubated with $0.5 \%$ Triton X-100 in PBS for $5 \mathrm{~min}$ and washed again three times with PBS. Afterwards, cells were added with $3 \%$ nonfat dry milk in PBS as blocking solution, incubated for $30 \mathrm{~min}$, and rinsed with PBS. Cells were incubated with phalloidin staining solution ( 5 units of phalloidin CF $488 \mathrm{~A} / \mathrm{ml}$ 1\% BSA-PBS, Linaris $\mathrm{GmbH}$, Dossenheim, Germany) for $20 \mathrm{~min}$ in the dark and washed three times with PBS. Cells were mounted with VECTASHIELD mounting medium with DAPI, stored at $4^{\circ} \mathrm{C}$, and protected from light. Phalloidin staining was analyzed by fluorescence microscopy with a Leica DMi8 microscope (Leica Microsystems, Wetzlar, Germany).
2.10. Statistical Analyses. Statistical analyses were performed using two-tailed unpaired or paired $t$-test, and $P$ values less than 0.05 were considered significant. All values were obtained from at least three technical replicates, except for qPCR analysis of murine mRNA which was performed in duplicate and expressed as mean \pm SD. Asterisks indicate significant differences against control samples used for normalization (dashed line), and hash sign indicates significant differences between samples (compared samples are annotated with connecting lines). Further details of the number of independent experiments, BMSC donors used, and selection of the normalization method are given in the figure legends.

\section{Results}

3.1. NOTCH Target Gene Activation in Osteocyte-Enriched Bone after In Vivo Tibial Mechanical Loading. To evaluate NOTCH activation after tibial mechanical loading in mice, we performed qPCR analysis of NOTCH target genes in osteocyte-enriched bone from left-loaded and right-nonloaded limbs [19]. 1 and $24 \mathrm{~h}$ after a single loading session, we detected up to 6-fold increases in mRNA expression of the NOTCH targets HES1, HEY1, and HEY2 dependent on the time point (Figures 1(a) and 1(b)).

3.2. NOTCH Signaling Is Activated in BMSCs after Cyclic Stretching. To clarify if NOTCH signaling is responsive for mechanical strain in osteogenic precursors, BMSCs from five donors were seeded on PU dishes and mechanical strain $(1 \mathrm{~Hz}, 1 \%)$ was applied. In donor \#02, gene expression of the NOTCH receptors NOTCH1 and NOTCH2 was induced over 60-fold and 30-fold, respectively; three donors (\#1, \#4, and \#5) responded with a 2 - to 3 -fold increase in NOTCH1 and NOTCH2 expression (Figure 2(a)). Additionally, expression of the NOTCH target genes HES1, HEY1, $H E Y 2$, and HEYL was quantified. Donor \#02 depicted the highest induction of HES1 (7-fold), HEY1 (9-fold), and HEYL (18-fold), whereas donor \#05 showed the highest increase in HEY2 expression (12-fold) after cyclic stretching. In two donors, expression of HEY1 (\#1 and \#5) and HEY2 (\#1 and \#2) was upregulated 2- to 3-fold, and in one donor, HES1 (\#5) and HEYL (\#4) gene expression was increased 3to 5 -fold. The NOTCH ligand JAG1 was also increased after mechanical stimulation. Donor \#02 depicted a 9-fold increase and donor \#05 a 4-fold increase. Analysis of the early osteogenic transcription factor RUNX2 revealed a more than ten-fold upregulation in donor \#02 and a 4-fold induction in donor \#01 and donor \#04 (Figure 2(b)). Overall, application of cyclic strain to BMSCs induced expression of NOTCH1; NOTCH2; the NOTCH ligand JAG1; the NOTCH target genes HES1, HEY1, HEY2, and HEYL; and the early osteogenic transcription factor RUNX2 with high donor variability.

To confirm that BMSCs are mechanosensitive and respond to cyclic stretching expression of the mechanoresponsive genes, PTGS2 and FOS [9,33] were analyzed in the same donors $15 \mathrm{~min}$ and $4 \mathrm{~h}$ after mechanical loading (Figure 2(c)). While PTGS2 was induced early after $15 \mathrm{~min}$ in donor \#03 and donor \#05, donor \#02 responded 
TABle 1: Primer names, sequences, product lengths, annealing temperatures, and GenBank accession numbers are shown.

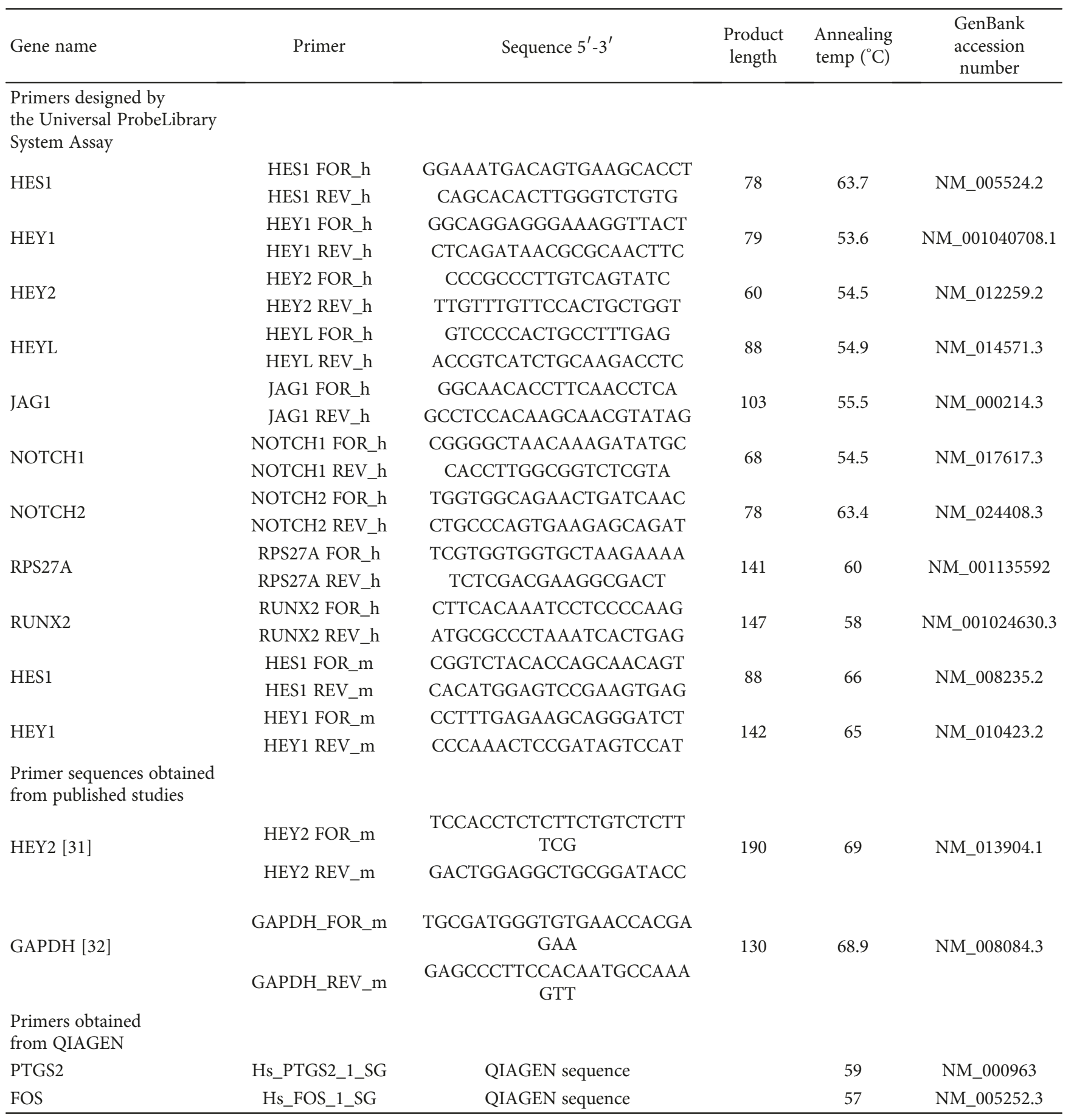

later at $4 \mathrm{~h}$. FOS gene expression was induced after $15 \mathrm{~min}$ in donors \#01, \#03, \#04, and \#05, while donor \#02 showed the highest induction $4 \mathrm{~h}$ after cyclic stretching. qPCR revealed a donor- and time-dependent induction of PTGS2 and FOS, while all donors responded to cyclic strain and were mechanoresponsive.

3.3. NOTCH Inhibition Does Not Affect Cell Viability and Apoptosis of BMSCs. In a next step, we blocked $\mathrm{NOTCH}$ signaling through the gamma-secretase inhibitor
GSI XII in BMSCs and hMSC-TERT cells. BMSCs and hMSC-TERT cells were treated with 2 and $4 \mu \mathrm{M}$ of GSI XII or the solvent DMSO $(0 \mu \mathrm{M})$. GSI XII doses were used for NOTCH inhibition as described previously [34]. Neither hMSC-TERT cells (Figure 3(a)) nor primary BMSCs (Figure 3(b)) showed a decrease in cell viability or an increase in apoptosis after 24 or $48 \mathrm{~h}$, respectively. Therefore, we determined GSI XII doses of 2 and $4 \mu \mathrm{M}$ as suitable for NOTCH inhibition without cell toxic side effects. 


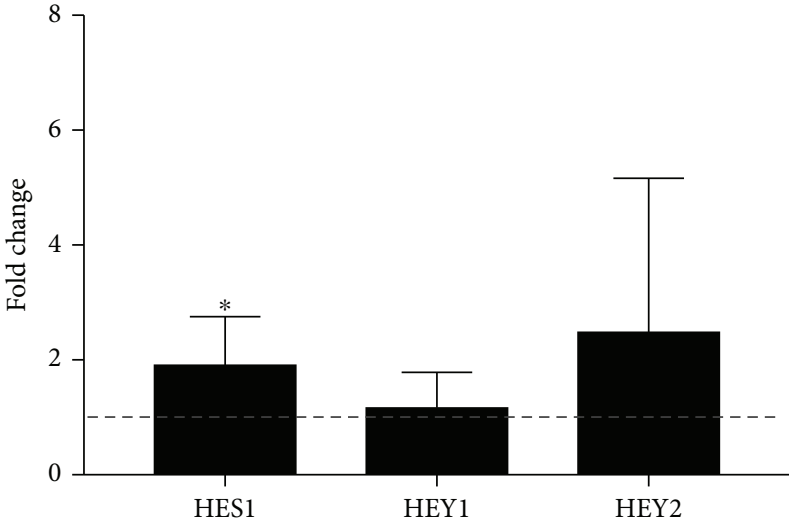

(a)

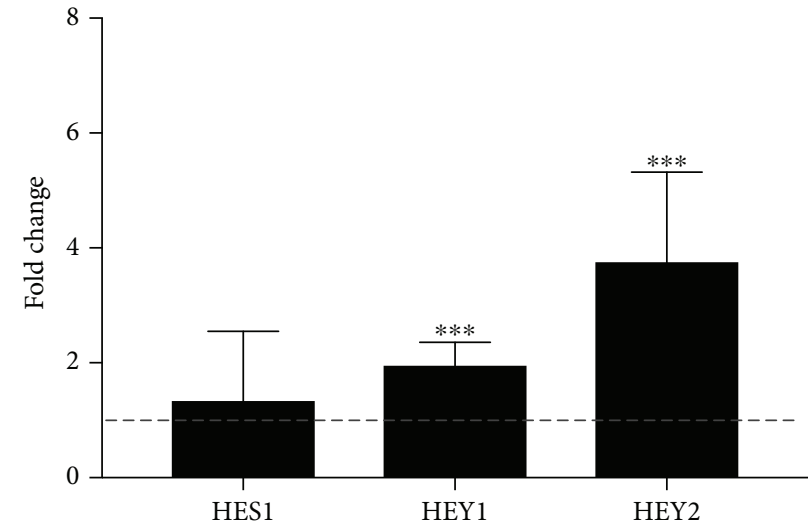

(b)

Figure 1: Gene expression of HES1, HEY1, and HEY2 1 and $24 \mathrm{~h}$ after a single loading session in left-loaded and right-nonloaded osteocyte-enriched tibiae of 10-week-old female C57BL/6 mice. Fold changes in gene expression of loaded limbs $(n=6)$ normalized to control limbs are shown (a) $1 \mathrm{~h}$ and (b) $24 \mathrm{~h}$ after loading. qPCR data were obtained from technical duplicates. Results are shown as mean $\pm \mathrm{SD}$; fold change was calculated with the $\Delta \Delta \mathrm{Ct}$ method and normalized to basal expression (nonloaded right limb, dashed line). GAPDH served as the housekeeping gene. Two-tailed paired $t$-test was used for statistical analysis $\left({ }^{*} P<0.05\right.$ and $\left.{ }^{* * *} P<0.001\right)$.
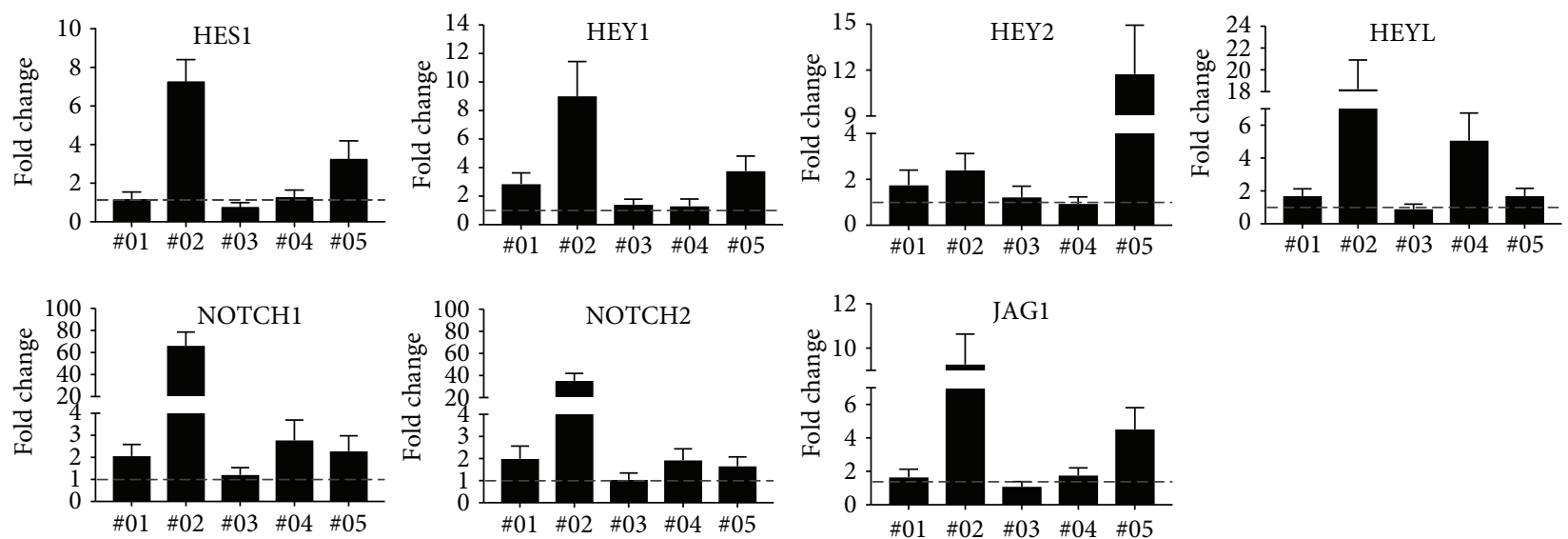

(a)

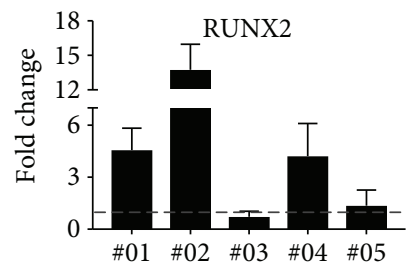

(b)
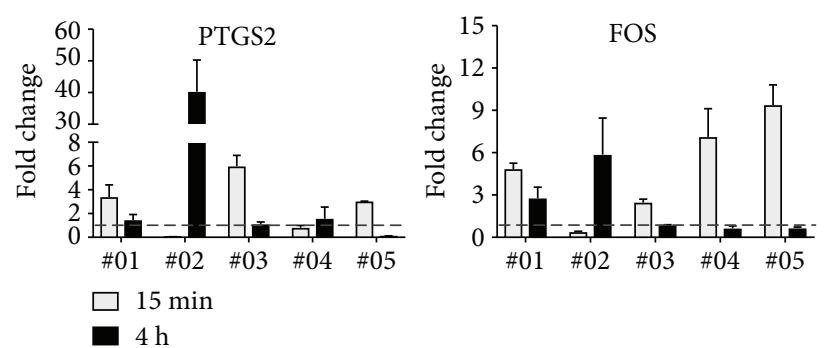

(c)

FIGURE 2: NOTCH target gene expression after cyclic stretching of BMSCs derived from five donors (donor \#01-donor \#05). (a) Relative mRNA expression of the NOTCH target genes HES1, HEY1, HEY2, and HEYL; the NOTCH1 and NOTCH2 receptor genes; and the NOTCH ligand gene JAG1. (b) Relative mRNA expression of the early osteogenic transcription factor RUNX2. Samples in (a) and (b) were collected $4 \mathrm{~h}$ after cyclic stretching. (c) Relative mRNA expression of the mechanoresponsive genes PTGS2 and FOS was analyzed $15 \mathrm{~min}$ and $4 \mathrm{~h}$ after cyclic stretching. qPCR data were obtained from technical triplicates $(\mathrm{a}, \mathrm{b})$ or from technical triplicates derived from three independent qPCR (c). Results are shown as mean $\pm \mathrm{SD}$; fold change was calculated with the $\Delta \Delta \mathrm{Ct}$ method and normalized to basal activity (nonstretched, dashed line). RPS27A served as the housekeeping gene.

3.4. NOTCH Inhibition Impairs Mechanotransduction in BMSCs and hMSC-TERT Cells. To evaluate if NOTCH inhibition has an impact on mechanotransduction in primary
BMSCs, cells were seeded on PU dishes and pretreated with 2 and $4 \mu \mathrm{M}$ GSI XII or the solvent DMSO for $24 \mathrm{~h}$ and mechanical strain was applied. Expression of the NOTCH 

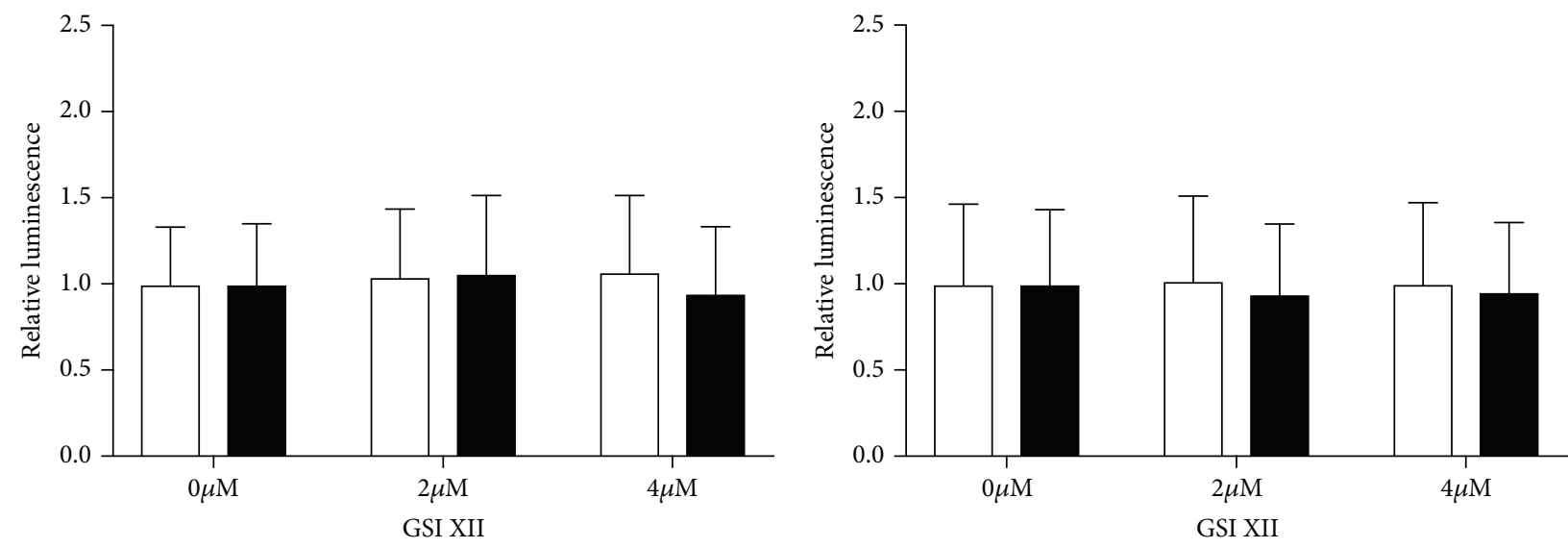

(a)
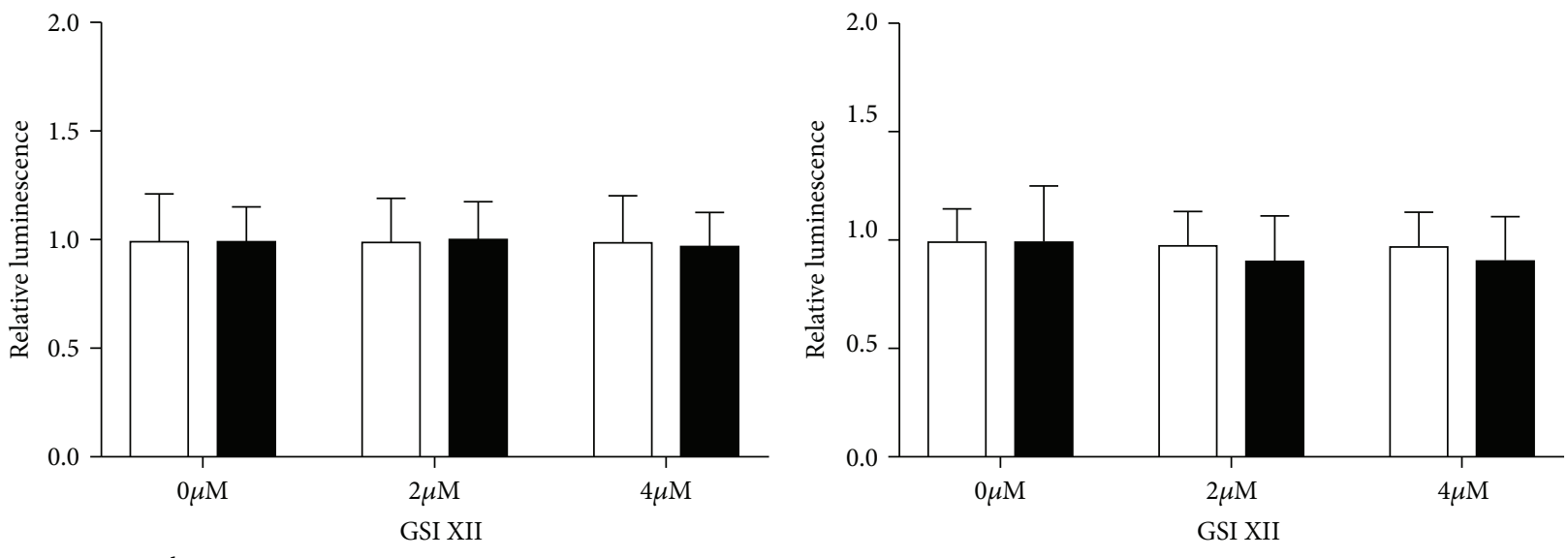

(b)

FIGURE 3: Effect of GSI XII on viability and apoptosis of hMSC-TERT cells and BMSCs derived from five donors (donor \#06-donor \#10). hMSC-TERT cells (a) and BMSCs (b) were treated with 2 and $4 \mu \mathrm{M}$ of GSI XII or the solvent DMSO $(0 \mu \mathrm{M})$, and viability and apoptosis assays were performed 24 and $48 \mathrm{~h}$ later. Relative luminescence is given. Data are expressed as mean of three independent experiments \pm SD (a) or mean of five different donors \pm SD (b) and normalized to untreated control. Each measurement was performed in technical triplicate. Two-tailed unpaired $t$-test was used for statistical analysis.

target genes $H E Y 1, H E Y 2$, and $H E Y L$ was analyzed by qPCR. Figure 4(a) shows results of four representative donors. In donor \#09, mechanodriven induction of HEY1 and HEYL $(0 \mu \mathrm{M}$ GSI XII) was blunted by GSI XII dose-dependently while expression of HEY2 was slightly influenced (Figure 4(a)). In donors \#10 and \#11, expression of HEY2 and HEYL was induced after cyclic stretching $(0 \mu \mathrm{M}$ GSI XII) and inhibited through GSI XII whereas HEY1 expression $(\# 12, \# 13)$ was not altered. In donor \#12, the NOTCH target HEY1 was upregulated ( $0 \mu \mathrm{M}$ GSI XII) 3 -fold after mechanical stimulation and no longer responded after NOTCH inhibition.

To analyze if the induction of a known and established mechanoresponsive gene in primary BMSCs could be reversed by NOTCH inhibition, expression of PTGS2 was analyzed by qPCR after pretreatment with GSI XII and application of cyclic strain. Mechanoresponse of PTGS2 was abolished significantly by GSI XII in a dose-dependent manner (Figure 4(b)).

By using hMSC-TERT, comprising a mechanoresponsive AP-1-driven luciferase reporter (hMSC-TERT-AP-1), we analyzed if NOTCH inhibition had an impact on the AP-1-mediated mechanoresponse. As reported before [22], AP-1-controlled luciferase activity was induced significantly by cyclic strain (Figure 4(c)). Induction of luciferase activity was abolished by 2 and $4 \mu \mathrm{M}$ GSI XII (black bars). No effect of GSI XII could be observed on hMSC-TERT-AP-1 cells that were grown without mechanical loading (white bars).

3.5. Phalloidin Staining Reveals a Cytoskeletal Modulation upon NOTCH Inhibition. We next investigated if cytoskeletal organization and actin remodeling, which mediate mechanotransduction in cells, are affected by NOTCH signaling at basal conditions (nonstretched). In untreated control BMSCs, 

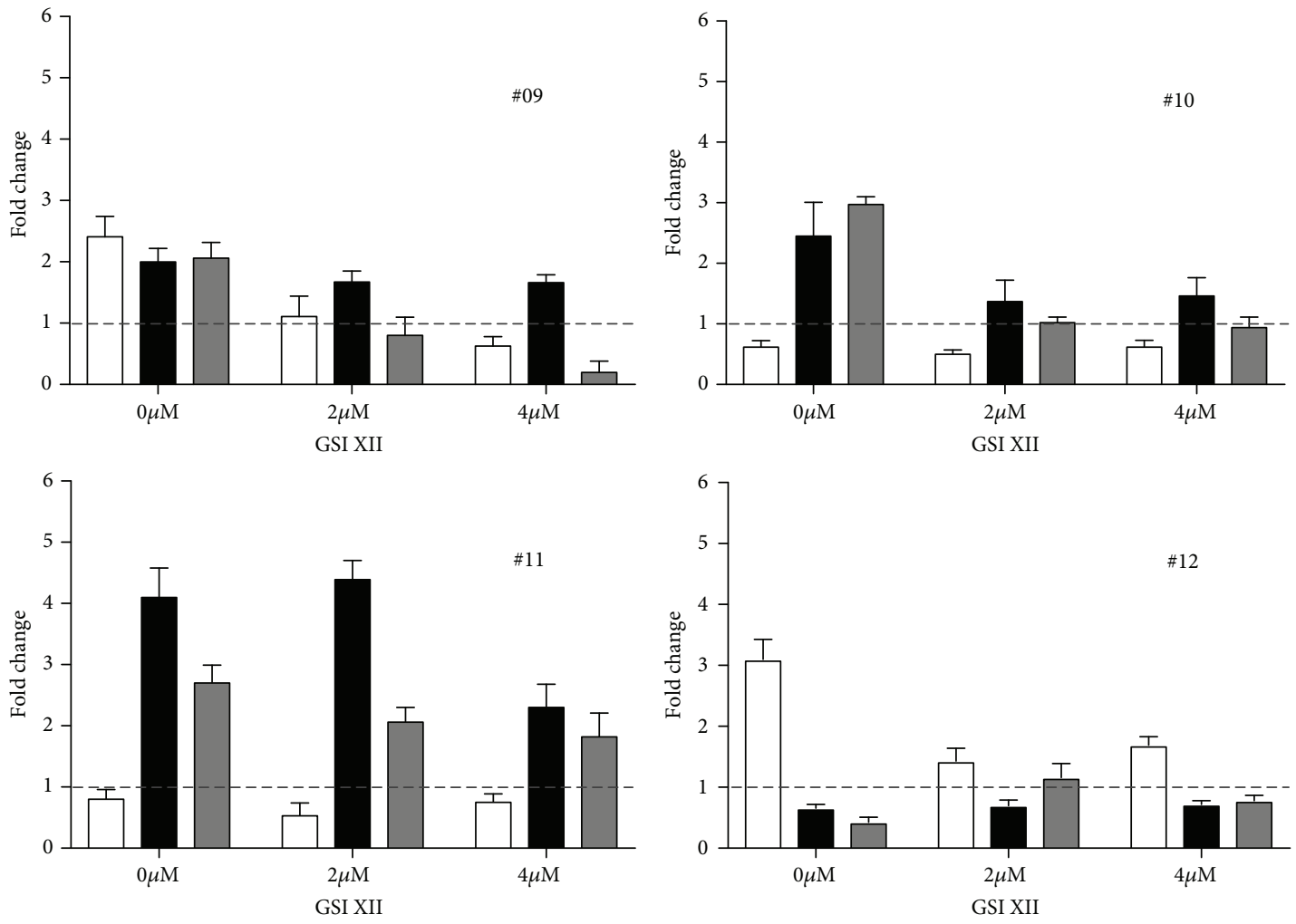

$\square$ HEY1
HEY2
$\square$ HEYL

(a)

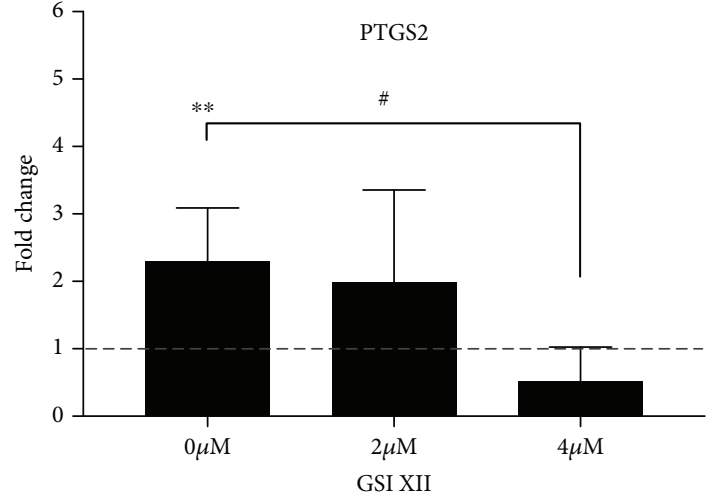

(b)

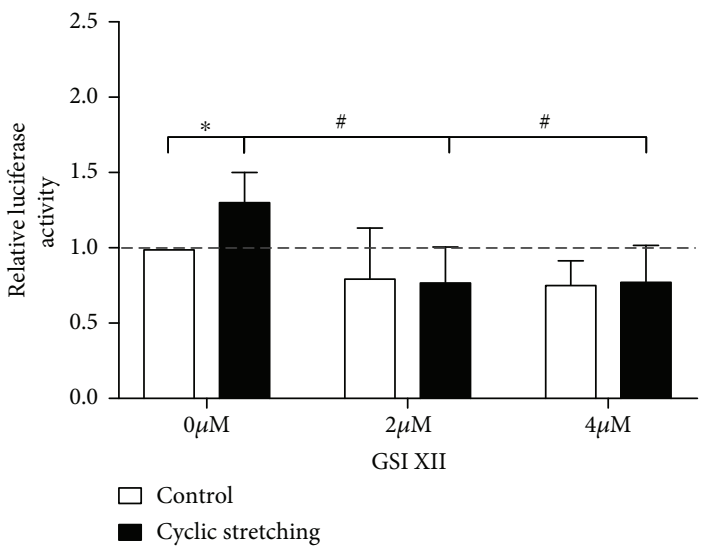

(c)

FIGURE 4: Effect of NOTCH inhibition on mechanotransduction (donor \#09-donor \#12). (a) Relative mRNA expression of the NOTCH target genes HEY1, HEY2, and HEYL in BMSCs from four representative donors after stretching and GSI inhibition as described in (b). Results are shown as mean of technical triplicates \pm SD and normalized to basal activity (DMSO treated, nonstretched, dashed line). Fold change was calculated with the $\triangle \triangle \mathrm{Ct}$ method, and RPS27A served as the housekeeping gene. (b) Relative mRNA expression of the PTGS2 gene in BMSCs. Cells were treated with $2 \mu \mathrm{M}$ and $4 \mu \mathrm{M}$ GSI XII or the solvent DMSO and received cyclic stretching. Samples were collected $4 \mathrm{~h}$ later. Results are shown as mean of four donors (\#09-12) \pm SD and normalized to basal activity (DMSO or GSI XII treated, nonstretched, dashed line). Fold change was calculated with the $\Delta \Delta \mathrm{Ct}$ method, and RPS27A served as the housekeeping gene. Two-tailed unpaired $t$-test was used for statistical analysis $\left({ }^{* *} P<0.01\right.$ : stretched compared to nonstretched, ${ }^{\#} P<0.01$ : DMSO control compared to $4 \mu \mathrm{M}$ GSI XII). (c) hMSC-TERT-AP-1 cells were treated with $2 \mu \mathrm{M}$ and $4 \mu \mathrm{M}$ GSI XII or DMSO as solvent and received cyclic stretching. Luciferase activity was determined and normalized to protein content. Data are expressed as mean of four independent experiments \pm SD and normalized to nonstretched, untreated controls (first column). Each measurement was performed in technical quadruplicate $\left({ }^{*} P<0.05\right.$ : stretched compared to nonstretched, \#P<0.05: DMSO control compared to $2 \mu \mathrm{M}$ or $4 \mu \mathrm{M}$ GSI XII). 


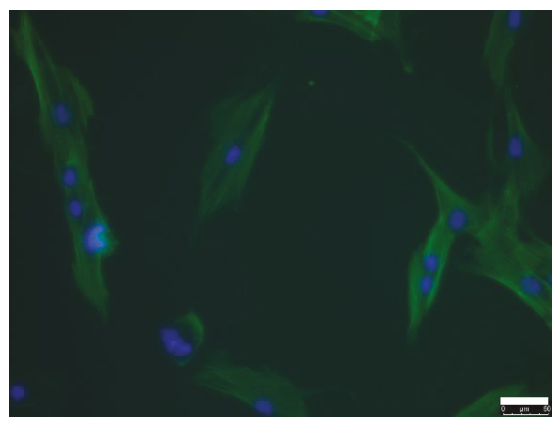

(a)

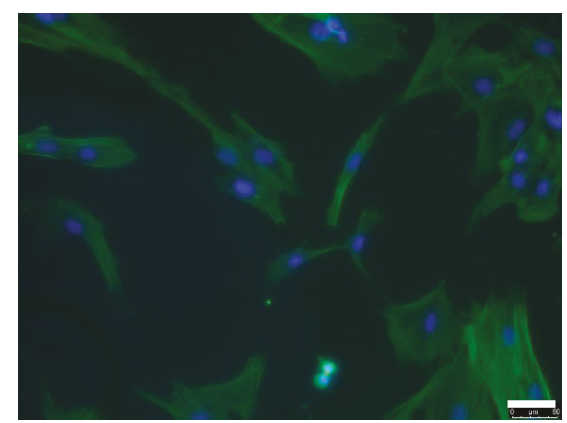

(b)

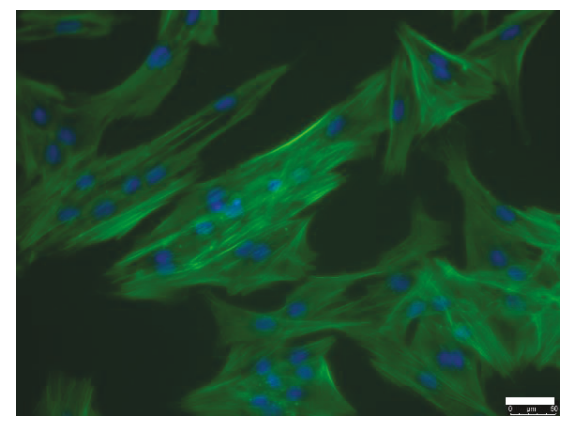

(c)

FIGURE 5: Phalloidin staining (green) of BMSCs after treatment with the NOTCH inhibitor GSI XII. Cells were incubated with $2 \mu \mathrm{M}$ (b) and $4 \mu \mathrm{M}$ (c) GSI XII for $24 \mathrm{~h}$; DMSO-treated cells served as a control (a). Representative images of one donor of three independent experiments are shown. DAPI (blue) was used for nuclear staining. The bar represents $50 \mu \mathrm{m}$.

phalloidin staining revealed a diffuse cytoskeleton organization (Figure 5(a)). After treatment of BMSCs with 2 and $4 \mu \mathrm{M}$ of GSI XII (Figures 5(b) and 5(c)) for 24h, the cytoskeleton depicted more structured actin fibers compared to untreated cells. In addition, GSI XII treatment resulted in a brighter phalloidin staining indicating a higher actin content or a denser and stiff arrangement of actin fibers.

3.6. The NOTCH2 Receptor Mediates NOTCH Signaling in hMSC-TERT Cells. Next, we investigated which NOTCH receptor (NOTCH1 or NOTCH2) mainly mediates signaling in BMSCs. We found that the transmembrane part of the NOTCH2 receptor (NTM) is expressed higher (up to 2-fold) than that of the NTM of NOTCH1 in hMSC-TERT-AP-1 cells (Figure 6(a)). Figure 6(a) shows a representative blot where densitometrical analysis of NTM normalized to the expression of the housekeeping protein vinculin was performed. To study specific NOTCH effects on nonstretched hMSC-TERT-AP-1 cells, we performed lentivirally mediated shRNA knockdown studies of NOTCH1 and NOTCH2 receptors (Figures $6(\mathrm{~b})$ and $6(\mathrm{~d})$ ). Efficient mRNA knockdown of NOTCH1 (shN1_1, shN1_2, Figure 6(b)) and NOTCH2 (shN2, Figure 6(d)) was confirmed by qPCR analysis. In addition, immunoblotting revealed reduction of NOTCH2 ( $\operatorname{sh} 2$ : 0.08-fold compared to ctr; normalized to vinculin) and HES1 (shN2: 0.76-fold compared to ctr; normalized to vinculin) protein levels after shRNA transfer (Figure 6(c)). We further evaluated the mRNA expression of NOTCH target (HES1, HEY1, and HES6) and mechanoresponsive (FOS, PTGS2) genes after specific NOTCH1 and NOTCH2 knockdown compared to control plasmid in
hMSC-TERT-AP-1 cells (Figures 6(b) and 6(d)). NOTCH2 knockdown revealed significant downregulation of the NOTCH target genes HES1 and HES6 and the mechanoresponsive genes FOS and PTGS2 (Figure 6(d)). NOTCH1 knockdown with two different shRNAs (shN1_1 and shN1_ 2) showed almost no significant effects on target and mechanoresponsive genes (Figure 6(b)).

\section{Discussion}

In this study, we evaluated expression of the NOTCH target genes HES1, HEY1, and HEY2 in murine tibial osteocyte-enriched bones after mechanical stimulation. No previous studies have reported NOTCH signaling after in vivo mechanical loading as much more attention has been focused on the WNT signaling pathway [35-38]. Our data indicate that NOTCH signaling is activated in bone cells as part of the anabolic mechanoresponse. We further evaluated whether osteoblastic precursors, mechanosensitive BMSCs, are a target of NOTCH activity in the bone. To that end, we used our small-scale cell culture/bioreactor system and applied cyclic stretching to analyze subcellular crosstalk mechanisms in mechanotransduction of human primary BMSCs and immortalized hMSC-TERT cells. In individual BMSC preparations, mRNA expression of NOTCH target genes such as HES1, HEY1, HEY2, and HEYL and the NOTCH1 and NOTCH2 genes and their ligand JAG1 was commonly increased after cyclic stretching although high donor variability was observed. To verify if all used BMSC donors responded to mechanical loading, we harvested RNA of BMSCs $15 \mathrm{~min}$ and $4 \mathrm{~h}$ after cyclic stretching and amplified 


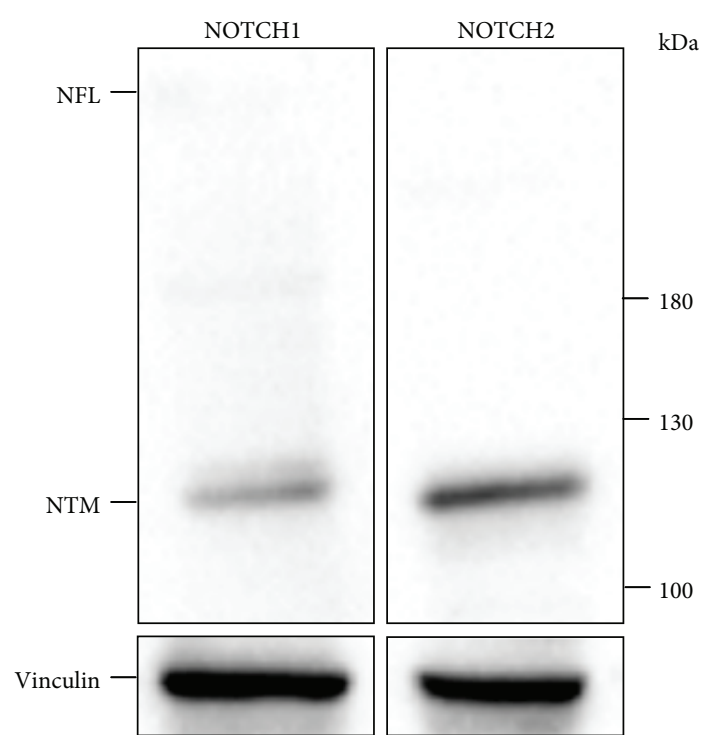

(a)

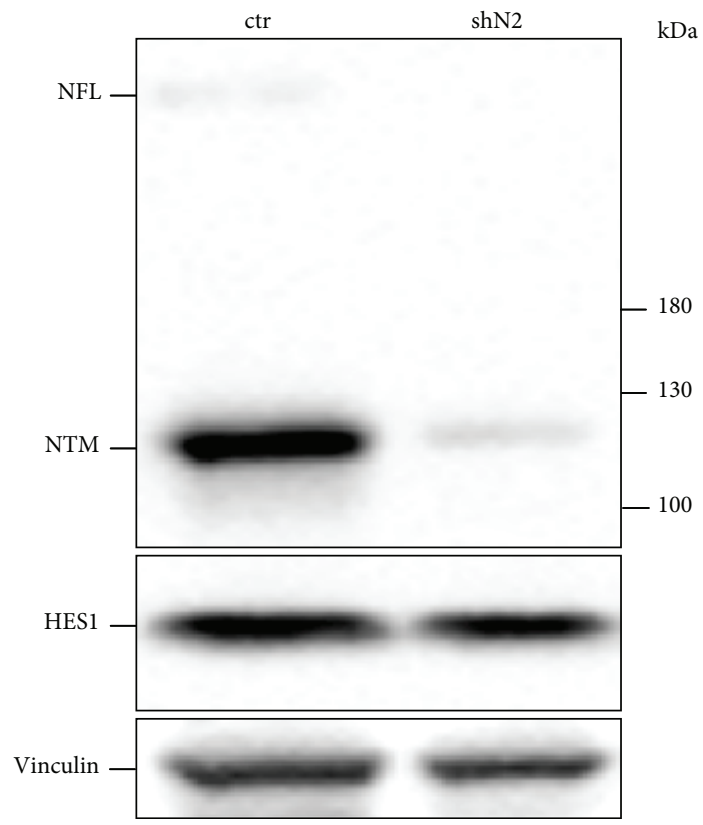

(c)

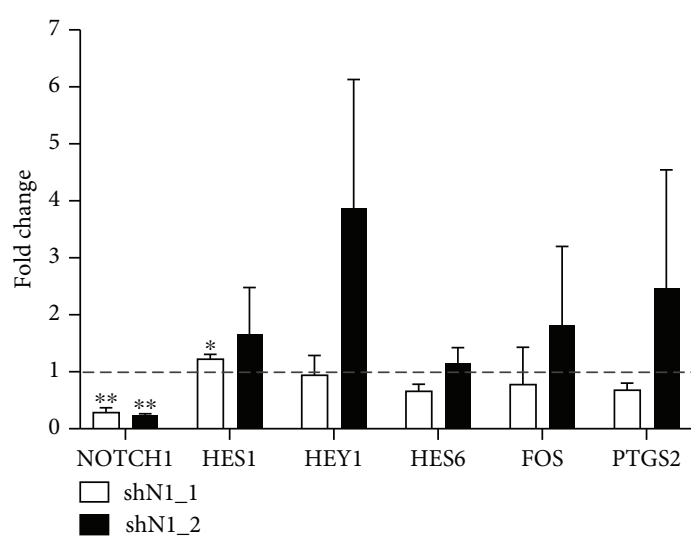

(b)

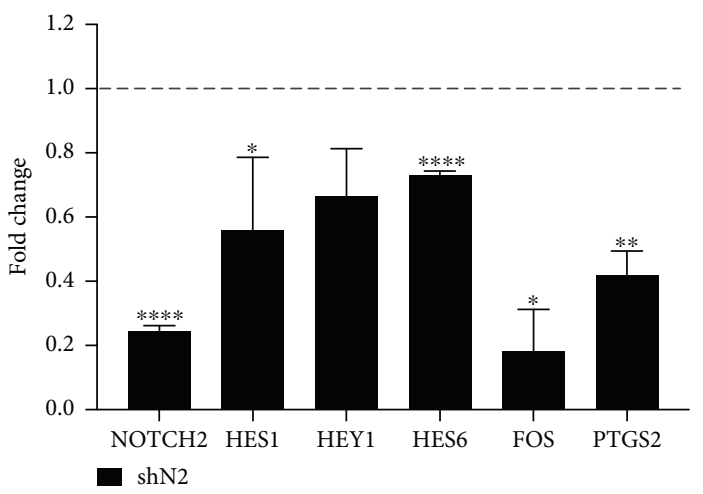

(d)

Figure 6: Knockdown of NOTCH1 and NOTCH2 genes in hMSC-TERT-AP-1 cells. (a) Immunoblotting of whole cell lysates of hMSC-TERT-AP-1 cells with antibodies against NOTCH1 and NOTCH2. Full-length NOTCH (NFL), transmembrane and intracellular part of NOTCH (NTM), and housekeeping protein vinculin. (b) hMSC-TERT-AP-1 cells were stably transduced with lentiviral vectors expressing shRNAs against NOTCH1 (shN1_1 and shN1_2). Relative mRNA expression of NOTCH1, HES1, HEY1, HES6, FOS, and PTGS2 is shown. Results are given as mean of two independent experiments $\pm \mathrm{SD}$ and normalized to an empty vector (dashed line). (c) Immunoblotting of whole cell lysates with antibodies against NOTCH2 after transfer of shN2 and nontargeting shRNA (ctr). (d) Relative mRNA expression of NOTCH2, HES1, HEY1, HES6, FOS, and PTGS2 after transfer of shN2 compared to nontargeting shRNA (dashed line). Two-tailed unpaired $t$-test was used for statistical analysis $\left({ }^{*} P<0.05,{ }^{* *} P<0.01\right.$, and $\left.{ }^{* * * *} P<0.0001\right)$. qPCR data were obtained from technical triplicates.

the known mechanoresponsive genes PTGS2 [33] and FOS [9]. As expected PTGS2 and FOS were upregulated after mechanical loading in all donors, dependent on the time point.

Inhibition of the NOTCH pathway in BMSC donors through GSI XII abolished the upregulation of NOTCH target genes after cyclic stretching. Moreover, upregulation of
PTGS2 after cyclic stretching in those BMSC donors and induction of the mechanosensitive AP-1-driven luciferase reporter in hMSC-TERT cells [22] were blocked by GSI XII in a dose-dependent manner. Our findings indicate that $\mathrm{NOTCH}$ signaling controls expression of mechanoresponsive genes in BMSCs. We therefore suggest that activation 
of the NOTCH pathway is part of the mechanotransduction process in BMSCs. To exclude cytotoxic effects of GSI XII on primary BMSCs or hMSC-TERT cells, we performed cell viability and apoptosis assays. No cell toxic effects of GSI XII were observed.

To clarify if NOTCH1, NOTCH2, or both receptors mediate the effects on BMSCs, we transduced hMSC-TERT-AP-1 cells with lentiviral knockdown constructs for NOTCH1 and $\mathrm{NOTCH} 2$. MRNA expression of NOTCH target genes and the mechanoresponsive FOS and PTGS2 genes were significantly downregulated in nonstretched hMSC-TERT cells after knockdown of NOTCH2. Our data reveal $\mathrm{NOTCH} 2$ as the key receptor, which mediates $\mathrm{NOTCH}$ effects on BMSCs.

The recent literature about the role of NOTCH signaling in bone biology favors a two-step model: (1) NOTCH signaling in early skeletal precursors inhibits osteogenic commitment and amplifies the precursor pool and (2) activation in later stages after osteoblast-to-osteocyte transition stimulates bone formation and mineralization via downregulation of the WNT inhibitors SOST and DKK1 and upregulation of the RANKL decoy receptor OPG [7]. Thereby, both precursor-specific and osteocyte-specific NOTCH activation may overcome age-associated osteopenia and prevent post-ovariectomy-induced bone loss. In the setting of fracture healing, NOTCH signaling promotes bone healing following osteotomy in mice [7]. It is well known that callus formation and fracture healing are markedly influenced by mechanical cues $[39,40]$. Our in vitro data provide a first functional link between NOTCH activation and the mechanoresponse of mechanosensitive skeletal precursors (BMSCs). A recent report described that in an osteoblast cell line, compressive forces enhanced $\mathrm{NOTCH}$ target gene expression via TGF $\beta$ signaling $[41,42]$. We suggest that BMSCs as precursors of osteoblasts and osteocytes contribute to the mechanoresponse of the early phases of bone regeneration and fracture healing through activation of NOTCH.

During mechanotransduction, extracellular signals are transduced via mechanoresponsive surface proteins from the outer cell membrane to the cytosol and the nucleus [43]. As the cytoskeleton is involved in this process and actin fibers are mediating the effects, we investigated if active NOTCH signaling is needed for proper cytoskeleton or actin organization. Interestingly, the actin cytoskeleton organization, as evidenced by phalloidin staining, was modulated through the NOTCH inhibitor GSI XII in a dose-dependent manner. We suggest that stiffening of actin fibers prevents mechanotransduction in BMSCs and that $\mathrm{NOTCH}$ activation is needed for cytoskeleton organization and function. Published data demonstrate a link between $\mathrm{NOTCH}$ signaling and cytoskeletal organization and actin remodeling in other cell types. In a zebrafish model, it has been shown that inhibition of NOTCH signaling led to a loss of stress fibers in the endoskeletal disc cells of the pectoral fin [44]. Overexpression of NIC in endothelial cells resulted in generation of stress fibers [45], and in hepatocellular carcinoma cells, application of actin cytoskeletal modulators resulted in activation of NOTCH signaling and induction of epithelial mesenchymal transition [46]. In BMSCs, our data indicate that active NOTCH signaling and the organization of the actin cytoskeleton are linked; however, the mechanism remains elusive.

Here, we present a first indication of how NOTCH signaling contributes to the complex network of transducing mechanosensitivity in BMSCs and we identified NOTCH2 as the key NOTCH receptor in BMSCs. Our findings provide a functional link between NOTCH activation and mechanotransduction in BMSCs as mediators of the anabolic response in bones.

\section{Conclusion}

NOTCH signaling is activated through mechanical strain in human BMSCs and after in vivo tibial mechanical loading in bone cells of wild-type mice. NOTCH controls expression of mechanoresponsive genes and is part of the mechanotransduction process in BMSCs.

\section{Data Availability}

The raw data of the $\mathrm{qPCR}$ and viability assays used to support the findings of this study are available from the corresponding author upon request.

\section{Conflicts of Interest}

There is no conflict of interest.

\section{Acknowledgments}

This work was supported by the Deutsche Forschungsgemeinschaft (DFG) grant JU 426 5-1 to F. Jundt, by the Bundesministerium für Bildung und Forschung (BMBF) grant 01EC1402C (DIMEOs) and by the Europäischer Fonds für regionale Entwicklung (EFRE) grant EU-1650-0006 to F. Jakob, and by the Deutsche Forschungsgemeinschaft (DFG, German Research Foundation)-Projektnummer 326998133-TRR 225 (subproject B05, R. Ebert). We thank Moustapha Kassem, Odense, Denmark, for supporting us with the hMSC-TERT cell line. We thank Catherine Julien for helping with the RT-qPCR.

\section{References}

[1] S. Zanotti and E. Canalis, "Notch signaling and the skeleton," Endocrine Reviews, vol. 37, no. 3, pp. 223-253, 2016.

[2] M. J. Hilton, X. Tu, X. Wu et al., "Notch signaling maintains bone marrow mesenchymal progenitors by suppressing osteoblast differentiation," Nature Medicine, vol. 14, no. 3, pp. 306314, 2008

[3] E. Canalis, D. J. Adams, A. Boskey, K. Parker, L. Kranz, and S. Zanotti, "Notch signaling in osteocytes differentially regulates cancellous and cortical bone remodeling," Journal of Biological Chemistry, vol. 288, no. 35, pp. 25614-25625, 2013.

[4] E. Canalis, K. Parker, J. Q. Feng, and S. Zanotti, "Osteoblast lineage-specific effects of Notch activation in the skeleton," Endocrinology, vol. 154, no. 2, pp. 623-634, 2013. 
[5] J. Shao, Y. Zhou, and Y. Xiao, "The regulatory roles of Notch in osteocyte differentiation via the crosstalk with canonical Wnt pathways during the transition of osteoblasts to osteocytes," Bone, vol. 108, pp. 165-178, 2018.

[6] X. Tu, J. Delgado-Calle, K. W. Condon et al., "Osteocytes mediate the anabolic actions of canonical Wnt/ $\beta$-catenin signaling in bone," Proceedings of the National Academy of Sciences of the United States of America, vol. 112, no. 5, pp. E478-E486, 2015.

[7] P. Liu, Y. Ping, M. Ma et al., "Anabolic actions of Notch on mature bone," Proceedings of the National Academy of Sciences of the United States of America, vol. 113, no. 15, pp. E2152E2161, 2016.

[8] C. Wang, J. Shen, K. Yukata et al., "Transient gamma-secretase inhibition accelerates and enhances fracture repair likely via Notch signaling modulation," Bone, vol. 73, pp. 77-89, 2015.

[9] A. Liedert, D. Kaspar, R. Blakytny, L. Claes, and A. Ignatius, "Signal transduction pathways involved in mechanotransduction in bone cells," Biochemical and Biophysical Research Communications, vol. 349, no. 1, pp. 1-5, 2006.

[10] S. Muller-Deubert, L. Seefried, M. Krug, F. Jakob, and R. Ebert, "Epidermal growth factor as a mechanosensitizer in human bone marrow stromal cells," Stem Cell Research, vol. 24, pp. 69-76, 2017.

[11] D. Pelaez, N. Arita, and H. S. Cheung, "Extracellular signal-regulated kinase (ERK) dictates osteogenic and/or chondrogenic lineage commitment of mesenchymal stem cells under dynamic compression," Biochemical and Biophysical Research Communications, vol. 417, no. 4, pp. 1286-1291, 2012.

[12] L. Seefried, S. Muller-Deubert, M. Krug et al., "Dissection of mechanoresponse elements in promoter sites of the mechanoresponsive CYR61 gene," Experimental Cell Research, vol. 354, no. 2, pp. 103-111, 2017.

[13] J. Lee, P. Fei, R. R. Packard et al., "4-Dimensional light-sheet microscopy to elucidate shear stress modulation of cardiac trabeculation," The Journal of Clinical Investigation, vol. 126, no. 5, pp. 1679-1690, 2016.

[14] V. C. Luca, B. C. Kim, C. Ge et al., "Notch-Jagged complex structure implicates a catch bond in tuning ligand sensitivity," Science, vol. 355, no. 6331, pp. 1320-1324, 2017.

[15] A. P. Kusumbe, S. K. Ramasamy, T. Itkin et al., "Age-dependent modulation of vascular niches for haematopoietic stem cells," Nature, vol. 532, no. 7599, pp. 380-384, 2016.

[16] J. J. Mack, T. S. Mosqueiro, B. J. Archer et al., "NOTCH1 is a mechanosensor in adult arteries," Nature Communications, vol. 8, no. 1, p. 1620, 2017.

[17] B. Marcet, B. Chevalier, C. Coraux, L. Kodjabachian, and P. Barbry, "MicroRNA-based silencing of Delta/Notch signaling promotes multiple cilia formation," Cell Cycle, vol. 10, no. 17, pp. 2858-2864, 2011.

[18] S. K. Ramasamy, A. P. Kusumbe, M. Schiller et al., "Blood flow controls bone vascular function and osteogenesis," Nature Communications, vol. 7, no. 1, article 13601, 2016.

[19] D. Pflanz, A. I. Birkhold, L. Albiol et al., "Sost deficiency led to a greater cortical bone formation response to mechanical loading and altered gene expression," Scientific Reports, vol. 7, no. 1, p. 9435, 2017.

[20] E. Fernandez-Rebollo, B. Mentrup, R. Ebert et al., "Human platelet lysate versus fetal calf serum: these supplements do not select for different mesenchymal stromal cells," Scientific Reports, vol. 7, no. 1, p. 5132, 2017.
[21] R. Ebert, P. Benisch, M. Krug et al., "Acute phase serum amyloid A induces proinflammatory cytokines and mineralization via Toll-like receptor 4 in mesenchymal stem cells," Stem Cell Research, vol. 15, no. 1, pp. 231-239, 2015.

[22] L. Seefried, S. Mueller-Deubert, T. Schwarz et al., "A small scale cell culture system to analyze mechanobiology using reporter gene constructs and polyurethane dishes," European Cells and Materials, vol. 20, pp. 344-355, 2010.

[23] B. M. Abdallah, M. Haack-Sorensen, J. S. Burns et al., "Maintenance of differentiation potential of human bone marrow mesenchymal stem cells immortalized by human telomerase reverse transcriptase gene despite [corrected] extensive proliferation," Biochemical and Biophysical Research Communications, vol. 326, no. 3, pp. 527-538, 2005.

[24] J. L. Simonsen, C. Rosada, N. Serakinci et al., "Telomerase expression extends the proliferative life-span and maintains the osteogenic potential of human bone marrow stromal cells," Nature Biotechnology, vol. 20, no. 6, pp. 592-596, 2002.

[25] C. Fellmann, T. Hoffmann, V. Sridhar et al., "An optimized microRNA backbone for effective single-copy RNAi," Cell Reports, vol. 5, no. 6, pp. 1704-1713, 2013.

[26] F. Jundt, I. Anagnostopoulos, R. Forster, S. Mathas, H. Stein, and B. Dorken, "Activated Notch1 signaling promotes tumor cell proliferation and survival in Hodgkin and anaplastic large cell lymphoma," Blood, vol. 99, no. 9, pp. 3398-3403, 2002.

[27] C. A. Schneider, W. S. Rasband, and K. W. Eliceiri, "NIH image to ImageJ: 25 years of image analysis," Nature Methods, vol. 9, no. 7, pp. 671-675, 2012.

[28] F. Jundt, K. S. Probsting, I. Anagnostopoulos et al., "Jagged1-induced Notch signaling drives proliferation of multiple myeloma cells," Blood, vol. 103, no. 9, pp. 3511-3515, 2004.

[29] M. W. Pfaffl, "A new mathematical model for relative quantification in real-time RT-PCR," Nucleic Acids Research, vol. 29, no. 9, pp. 45e-445, 2001.

[30] Y. Kosaka, K. Mimori, T. Fukagawa et al., "Identification of the high-risk group for metastasis of gastric cancer cases by vascular endothelial growth factor receptor-1 overexpression in peripheral blood," British Journal of Cancer, vol. 96, no. 11, pp. 1723-1728, 2007.

[31] D. Xu, J. Hu, S. Xu et al., "Dll1/Notch activation accelerates multiple myeloma disease development by promoting CD138 + MM-cell proliferation," Leukemia, vol. 26, no. 6, pp. 14021405, 2012.

[32] J. Huang, Y. H. Hsu, C. Mo et al., "METTL21C is a potential pleiotropic gene for osteoporosis and sarcopenia acting through the modulation of the NF- $\kappa \mathrm{B}$ signaling pathway," Journal of Bone and Mineral Research, vol. 29, no. 7, pp. 1531-1540, 2014.

[33] A. Ogasawara, T. Arakawa, T. Kaneda et al., "Fluid shear stress-induced cyclooxygenase- 2 expression is mediated by $\mathrm{C} / \mathrm{EBP} \beta$, cAMP-response element-binding protein, and AP-1 in osteoblastic MC3T3-E1 cells," Journal of Biological Chemistry, vol. 276, no. 10, pp. 7048-7054, 2001.

[34] R. Schwarzer, B. Dorken, and F. Jundt, "Notch is an essential upstream regulator of NF- $\kappa \mathrm{B}$ and is relevant for survival of Hodgkin and Reed-Sternberg cells," Leukemia, vol. 26, no. 4, pp. 806-813, 2012.

[35] N. H. Kelly, J. C. Schimenti, F. P. Ross, and M. C. van der Meulen, "Transcriptional profiling of cortical versus cancellous bone from mechanically-loaded murine tibiae reveals differential gene expression," Bone, vol. 86, pp. 22-29, 2016. 
[36] A. Liedert, L. Wagner, L. Seefried, R. Ebert, F. Jakob, and A. Ignatius, "Estrogen receptor and Wnt signaling interact to regulate early gene expression in response to mechanical strain in osteoblastic cells," Biochemical and Biophysical Research Communications, vol. 394, no. 3, pp. 755-759, 2010.

[37] S. M. Mantila Roosa, Y. Liu, and C. H. Turner, "Gene expression patterns in bone following mechanical loading," Journal of Bone and Mineral Research, vol. 26, no. 1, pp. 100-112, 2011.

[38] G. Zaman, L. K. Saxon, A. Sunters et al., "Loading-related regulation of gene expression in bone in the contexts of estrogen deficiency, lack of estrogen receptor alpha and disuse," Bone, vol. 46, no. 3, pp. 628-642, 2010.

[39] L. E. Claes, C. A. Heigele, C. Neidlinger-Wilke et al., "Effects of mechanical factors on the fracture healing process," Clinical Orthopaedics and Related Research, vol. 355S, pp. S132-S147, 1998.

[40] B. Kruck, E. A. Zimmermann, S. Damerow et al., "Sclerostin neutralizing antibody treatment enhances bone formation but does not rescue mechanically induced delayed healing," Journal of Bone and Mineral Research, vol. 33, no. 9, pp. 1686-1697, 2018.

[41] Y. Ji, Y. Ke, and S. Gao, "Intermittent activation of Notch signaling promotes bone formation," American Journal of Translational Research, vol. 9, no. 6, pp. 2933-2944, 2017.

[42] J. Manokawinchoke, P. Pavasant, and T. Osathanon, "Intermittent compressive stress regulates Notch target gene expression via transforming growth factor- $\beta$ signaling in murine pre-osteoblast cell line," Archives of Oral Biology, vol. 82, pp. 47-54, 2017.

[43] F. Martino, A. R. Perestrelo, V. Vinarsky, S. Pagliari, and G. Forte, "Cellular mechanotransduction: from tension to function," Frontiers in Physiology, vol. 9, p. 824, 2018.

[44] S. Pascoal, J. Esteves de Lima, J. D. Leslie, S. M. Hughes, and L. Saude, "Notch signalling is required for the formation of structurally stable muscle fibres in zebrafish," PLoS One, vol. 8, no. 6, article e68021, 2013.

[45] E. Wieland, J. Rodriguez-Vita, S. S. Liebler et al., "Endothelial Notch1 activity facilitates metastasis," Cancer Cell, vol. 31, no. 3, pp. 355-367, 2017.

[46] J. M. Peng, R. Bera, C. Y. Chiou et al., "Actin cytoskeleton remodeling drives epithelial-mesenchymal transition for hepatoma invasion and metastasis in mice," Hepatology, vol. 67, no. 6, pp. 2226-2243, 2018. 


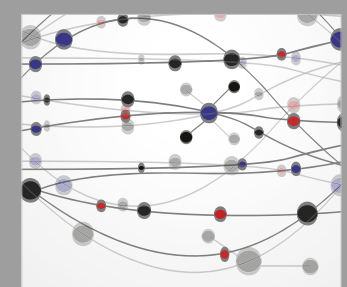

The Scientific World Journal
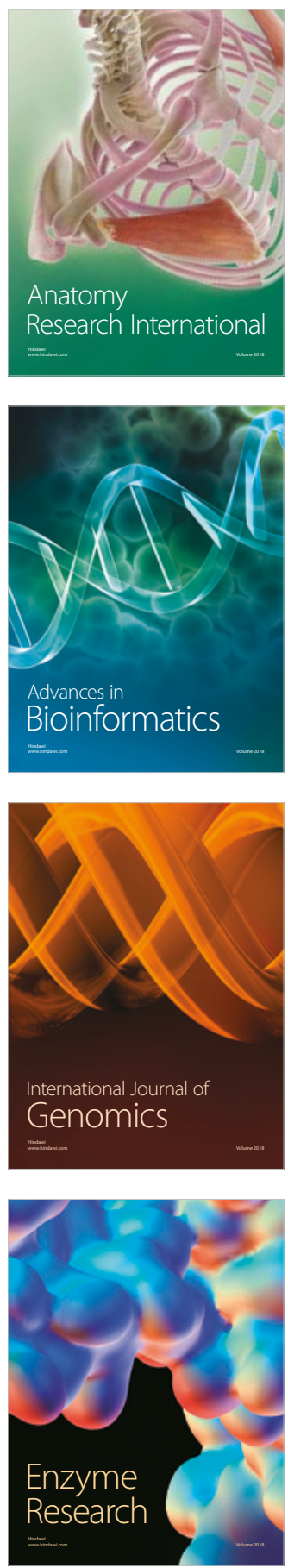
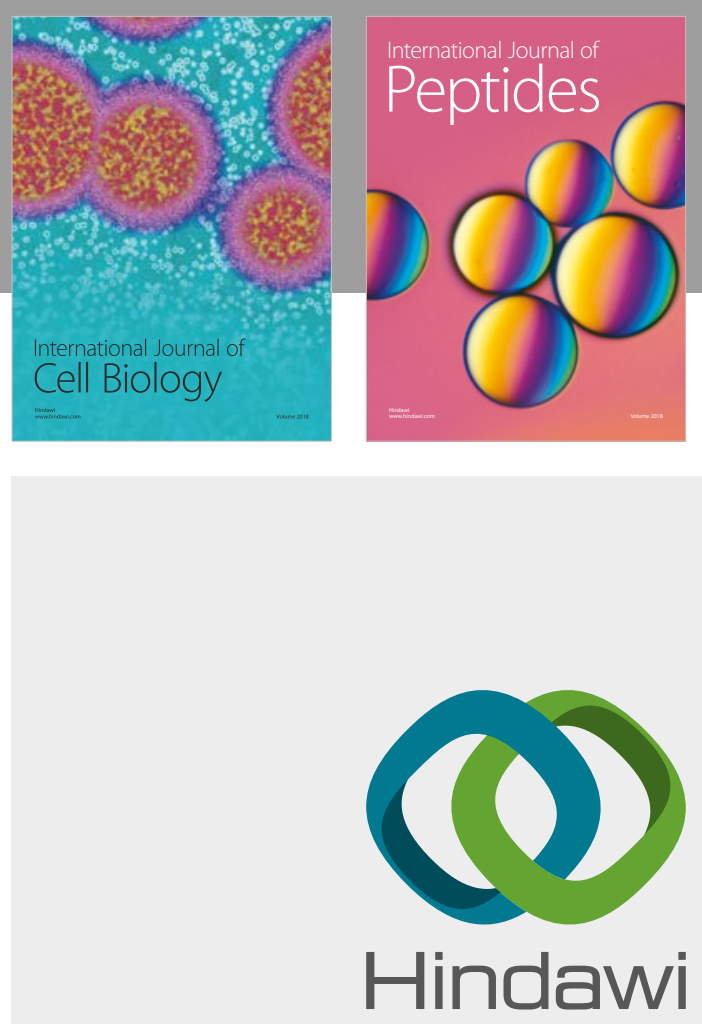

Submit your manuscripts at

www.hindawi.com
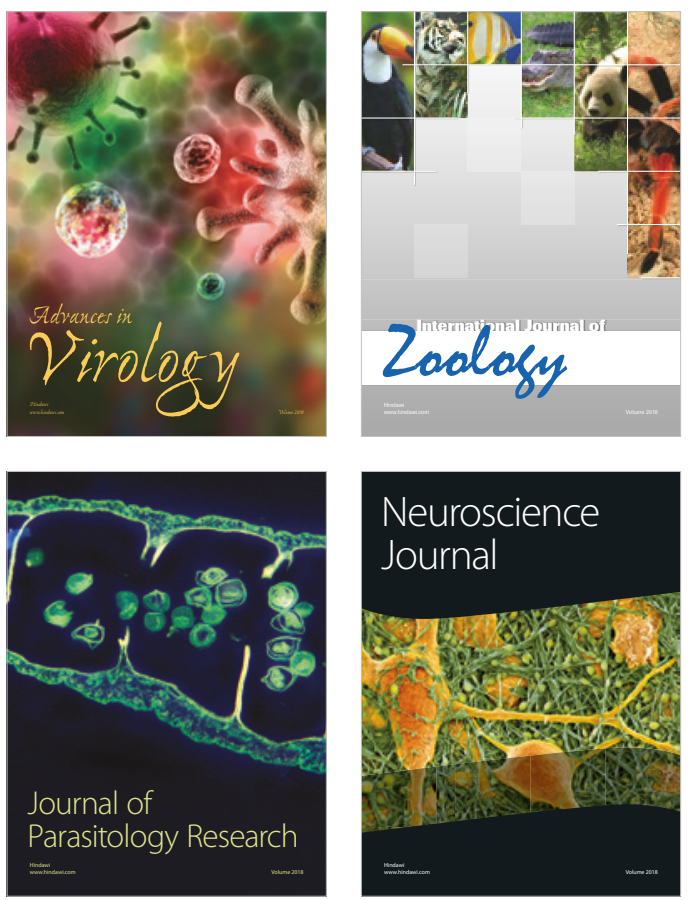
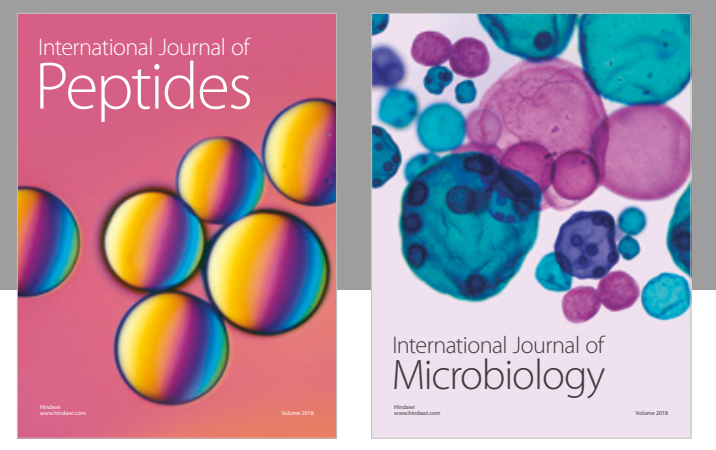

nternational Journal of Microbiology
Journal of
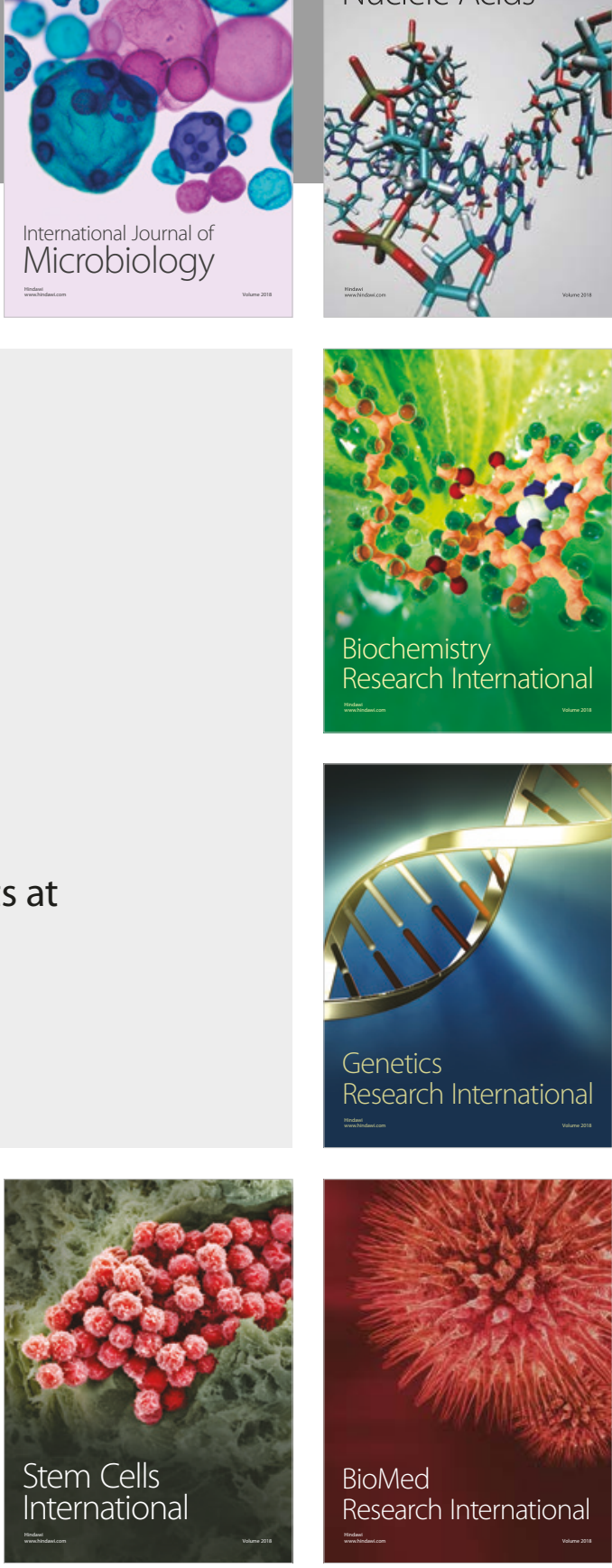
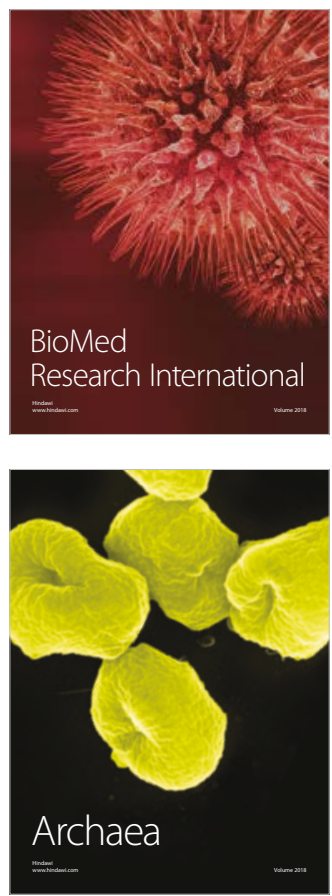